\title{
Nt-proANP in plasma, a marker of salt sensitivity, is reduced in type 2 diabetes patients
}

\author{
O. MELANDER ${ }^{1}$, E. FRANDSEN ${ }^{2}$, M. MAGNUSSON ${ }^{3}$, A. GRUBB ${ }^{4}$, S. JOVINGE \& L. GROOP $^{1}$ \\ From the ${ }^{1}$ Department of Endocrinology, Malmö University Hospital, Lund University, Malmö, Sweden, ${ }^{2}$ Department of Clinical Physiology and \\ Nuclear Medicine, Glostrup Hospital, Glostrup, Denmark, ${ }^{3}$ Department of Cardiology, Malmö University Hospital, Lund University, Malmö, and \\ ${ }^{4}$ Department of Clinical Chemistry, University Hospital, Lund, Sweden
}

\begin{abstract}
Melander O, Frandsen E, Magnusson M, Grubb A, Jovinge S, Groop L (Malmö University Hospital, Lund University, Malmö, Sweden; Glostrup Hospital, Glostrup, Denmark; and University Hospital, Lund, Sweden). Nt-proANP in plasma, a marker of salt sensitivity, is reduced in type 2 diabetes patients. J Intern Med 2005; 257: 281-288.
\end{abstract}

Objective. We recently showed that plasma concentration of N-terminal atrial natriuretic peptide (Nt-proANP) is strongly directly related to salt sensitivity. The aims of the present study were to test (i) whether plasma concentration of $\mathrm{N}$-terminal brain natriuretic peptide (Nt-proBNP) is related to salt sensitivity and (ii) whether Nt-proANP, as a marker of salt sensitivity, differs between type 2 diabetes patients and nondiabetic subjects without a history of coronary heart disease.

Methods. Nt-proBNP was determined in 30 Swedish normal subjects with heredity for primary hypertension and salt sensitivity was defined as the difference between mean arterial blood pressure after 1 week on a high-salt diet $\left(240 \mathrm{mmol} \mathrm{day}^{-1}\right)$ and 1 week on a low-salt diet $\left(10 \mathrm{mmol} \mathrm{day}^{-1}\right)$.
Nt-proANP was measured in 253 patients with type 2 diabetes and in 230 nondiabetic subjects aged 40-70 years, all without a history of coronary heart disease.

Results. Amongst the 30 subjects, in whom salt sensitivity was directly measured, Nt-proBNP was not correlated with salt sensitivity $(R=-0.18, P=$ 0.35). Nt-proANP (median, interquartile range) was lower in patients with type 2 diabetes $(505,387-$ $\left.661 \mathrm{pmol} \mathrm{L}^{-1}\right)$ than in nondiabetic subjects $(536$, 421-696 pmol L $\left.{ }^{-1}\right) \quad(P=0.02)$. In a multiple regression analysis heart rate $(P<0.00001)$, diastolic blood pressure $(P=0.02)$ and diabetes status $(P=0.02)$ were inversely related whereas age $(P<0.00001)$, cystatin $C \quad(P=0.0006)$, hypertension treatment $(P=0.002)$ and female sex $(P=0.006)$ were directly related to $\ln (\mathrm{Nt}$-proANP $)$. Conclusion. In contrast to Nt-proANP, Nt-proBNP is not related to salt sensitivity. Salt sensitivity, as estimated by Nt-proANP, seems to be reduced in type 2 diabetes.

Keywords: hypertension, N-terminal atrial natriuretic peptide, salt sensitivity, type 2 diabetes.

\section{Introduction}

As a consequence of new guidelines suggesting lower blood pressure limits for the diagnosis of hypertension and lower blood pressure treatment goals $[1,2]$, a substantial proportion of the general population will be considered for antihypertensive treatment. The majority of these patients have mild hypertension, a category well suited for nonpharmacological antihypertensive treatment alone or in combination with pharmacological treatment. Moderate dietary salt restriction is effective in reducing blood pressure in patients with mild hypertension in both Blacks and Whites [3]. As the majority of the ingested salt is added during industrial food processing, whereas salt added at the table and during cooking constitutes a minor source [4], a successful dietary low-salt intervention requires careful education of the patients of which products can be used. In addition, the blood pressure-lowering effect of salt restriction, i.e. the degree of salt sensitivity, varies substantially between individuals [5]. As salt sensitivity testing is expensive and cumbersome to perform in clinical practice, a surrogate marker of 
salt sensitivity would be of great value in order to focus treatment with dietary salt restriction to those patients who are likely to benefit the most from this intervention.

The 126-amino acid peptide atrial natriuretic peptide pro-hormone (proANP ${ }_{1-126}$ ) is synthesized and stored in atrial myocytes [6]. Upon distension of the cardiac atria, proANP ${ }_{1-126}$ is cleaved and equimolar amounts of the C-terminal fragment proANP $_{99-126}$ (ANP) and an N-terminal fragment (proANP $1-98$ ) are secreted from the atria. The $\mathrm{N}$-terminal fragment is subsequently cleaved into smaller peptide fragments [7], which have natriuretic, diuretic, blood pressure-lowering and kaliuretic properties [7, 8]. ANP is a potent mediator of natriuresis and vasodilatation [9]. Whereas ANP is rapidly removed from the circulation [10], the $\mathrm{N}$-terminal fragments, such as the proANP ${ }_{1-30}$ fragment (Nt-proANP), which is also termed long-acting natriuretic peptide and has strong salt-excreting properties itself $[7,8]$, are stable and remain in the circulation at manifold higher concentrations than ANP [11]. Nt-proANP measured in peripheral plasma is therefore less prone to fluctuation and may thus be a more reliable measure of atrial ANP secretion than peripheral plasma concentration of ANP itself. We recently showed that in subjects with heredity for hypertension, Nt-proANP is strongly related to salt intake and to degree of salt sensitivity (whether or not salt intake had been standardized when Nt-proANP was measured) [5], suggesting that Nt-proANP could be used as a surrogate marker of salt sensitivity.

Brain natriuretic peptide is produced in the ventricles by cleavage of its pro-hormone (proB$\mathrm{NP}_{1-108}$ ) to the biologically active $\mathrm{C}$-terminal fragment proBNP ${ }_{77-108}$ (BNP) and a biologically inactive $\mathrm{N}$-terminal fragment corresponding to the first 76 amino acids of proBNP ${ }_{1-108}$ (Nt-proBNP) [11]. Many of the actions of BNP are similar to those of ANP, and it has been suggested that Nt-proBNP, BNP and Nt-proANP are better in detecting early congestive heart failure when compared with ANP, but there is controversy in the literature as to which one of Nt-proBNP, BNP or Nt-proANP is most useful as marker for early congestive heart failure [12-14]. However, the relation between Nt-proBNP and salt sensitivity is not known. The first aim of the present study was therefore to examine whether Nt-proBNP, like Nt-proANP, is related to the degree of salt sensitivity.
Hypertension is present in as much as $70 \%$ of patients with type 2 diabetes, thus being considerably more common than in the nondiabetic population [15]. In addition, blood pressure reduction beyond what is recommended in nondiabetics has proved fruitful for prevention of cardiovascular disease in hypertensive patients with type 2 diabetes $[1,2]$. The reason for the increased prevalence of hypertension in type 2 diabetes is unknown. One of the main hypotheses is that hyperinsulinaemia, secondary to peripheral insulin resistance, causes sodium retention in the kidney that in turn could lead to salt-sensitive hypertension [16]. The second aim of this study was to investigate whether the degree of salt sensitivity differs between patients with type 2 diabetes and nondiabetic subjects, neither of the two groups having a history of coronary heart disease. As a surrogate measure of the degree of salt sensitivity, we used the plasma concentration of Nt-proANP [5].

\section{Method}

\section{Subjects and phenotyping}

The study was approved by the ethics committee of Lund University. The subject description and salt sensitivity phenotyping procedure of the 30 subjects in whom Nt-proBNP was related to the degree of salt sensitivity have been described in detail previously [5]. In brief, 13 men and 17 women, who had at least one first-degree relative with primary hypertension but were free from medication, diabetes and other chronic diseases, were investigated at baseline and after 1 and 2 weeks. After the baseline investigation, the study subjects were given a low-salt diet (10 mmol sodium and $70 \mathrm{mmol}$ potassium per day) for 1 week. During the second week, sodium chloride capsules $\left(230 \mathrm{mmol} \mathrm{day}^{-1}\right)$ were added to the diet to achieve a high salt intake of $240 \mathrm{mmol} \mathrm{day}{ }^{-1}$. Urine samples $(24 \mathrm{~h})$ were collected before the baseline investigation and at the end of the high- and low-salt diet weeks. The diet was prepared by a dietician and the daily energy intake was adjusted according to body weight and gender (8400-11 $760 \mathrm{~kJ})$. The study subjects received all meals and drinks from a metabolic ward and were not allowed to eat or drink anything else during the study period. Salt sensitivity was defined as the difference between mean arterial blood pressure (diastolic blood pressure plus 
one-third of the pulse pressure) after the high- $\left(240 \mathrm{mmol} \mathrm{day}{ }^{-1}\right)$ and low- $\left(10 \mathrm{mmol} \mathrm{day}{ }^{-1}\right)$ salt diet weeks. The study subjects' mean $( \pm S \mathrm{SD})$ age was $48.1 \pm 6.7$ years, body mass index (BMI) $26.8 \pm 3.6 \mathrm{~kg} \mathrm{~m}^{-2}$ and baseline systolic and diastolic blood pressure $135 \pm 12 \mathrm{mmHg}$ and $83.4 \pm$ $7.3 \mathrm{mmHg}$. Fasting plasma samples for analysis of Nt-proBNP were drawn in the morning under baseline conditions, i.e. before salt intake had been standardized [5].

In order to test whether Nt-proANP, as a marker of degree of salt sensitivity, is affected by the presence of type 2 diabetes, we studied 253 patients with type 2 diabetes and 230 nondiabetic subjects who were recruited from primary healthcare centres in the Botnia region in western Finland and in southern Sweden [17]. Type 2 diabetes was diagnosed according to World Health Organization criteria from 1985 [18] and the age at onset of the disease ranged between 40 and 66 years of age. All nondiabetic subjects had normal glucose tolerance according to an oral glucose tolerance test [18] and none had any first-degree relative with type 2 diabetes. All subjects underwent a physical examination and a standardized health questionnaire, with special emphasis on cardiovascular disease, was completed by specially trained nurses, as previously described [19]. Fasting blood samples were drawn for analysis of Nt-proANP, creatinine, cystatin $\mathrm{C}$ and glycated haemoglobin (HbAlc). To avoid disease states that may cause elevation in Nt-proANP independently of salt sensitivity, such as heart disease [12] and renal failure [20], the inclusion criteria in the present study were (i) no history of coronary heart disease [19], (ii) no use of digitalis or nitrates and (iii) serum creatinine $<140 \mu \mathrm{mol} \mathrm{L}{ }^{-1}$. As Nt-proANP is eliminated from the blood by glomerular filtration we measured plasma concentration of cystatin $\mathrm{C}$, a more sensitive marker of glomerular filtration rate (GFR) than serum creatinine [21], in order to be able to take into account differences in GFR within the range of serum creatinine levels of the study subjects.

Blood pressure and heart rate were measured twice in the right arm in the seated position after $30 \mathrm{~min}$ rest and the mean value of the two recordings was calculated. Korotkoff sounds corresponding to 'phase I' was used to define the systolic and 'phase V' the diastolic blood pressure. BMI was calculated after body weight and height were measured with subjects in light clothing without shoes. The clinical characteristics of the study subjects are shown in Table 1.

\section{Biochemical assays}

Plasma Nt-proBNP was measured using a commercial enzyme immunoassay (Biomedica, Wien, Austria). Plasma Nt-proANP was measured as described previously [5]. HbA1c concentration in the blood was measured by high-pressure liquid chromatography and serum creatinine was analysed with a kinetic method (Kone Oy Reag, Espoo, Finland). Cystatin C in plasma was measured by a fully automated particle-enhanced turbidimetric assay on undiluted samples [22, 23] using a Behring BN ProSpec analyser (Dade Behring, Marburg, Germany) and a calibrator (DakoCytomation,
Table 1 Clinical characteristics of nondiabetic subjects and type 2 diabetes patients with no history of coronary heart disease

\begin{tabular}{lllc}
\hline & $\begin{array}{l}\text { Nondiabetic } \\
\text { subjects } \\
(n=230)\end{array}$ & $\begin{array}{l}\text { Type 2 diabetes } \\
\text { patients } \\
(n=253)\end{array}$ & P-value \\
\hline Variable & $58.0 \pm 6.7$ & $58.6 \pm 6.3$ & 0.25 \\
Age (years) & 60.9 & 51.4 & 0.04 \\
Sex $(\%$ females) & $26.5 \pm 4.2$ & $29.0 \pm 5.0$ & $<0.00001$ \\
Body mass index $\left(\mathrm{kg} \mathrm{m}^{-2}\right)$ & $134 \pm 19.7$ & $145 \pm 18.3$ & $<0.00001$ \\
Systolic blood pressure $(\mathrm{mmHg})$ & $81.4 \pm 9.9$ & $83.8 \pm 10.0$ & 0.007 \\
Diastolic blood pressure $(\mathrm{mmHg})$ & 12.6 & 40.3 & $<0.00001$ \\
Antihypertensive treatment $(\%)$ & $63.4 \pm 14.2$ & $69.5 \pm 13.5$ & $<0.00001$ \\
Heart rate $\left(\mathrm{b}\right.$ min $\left.^{-1}\right)$ & $86.8 \pm 13.8$ & $85.9 \pm 15.1$ & 0.35 \\
Creatinine $\left(\mu \mathrm{mol} \mathrm{L}^{-1}\right)$ & $1.14 \pm 0.21$ & $1.11 \pm 0.24$ & 0.03 \\
Cystatin C $\left(\mu \mathrm{mol} \mathrm{L}^{-1}\right)$ & $5.3 \pm 0.42$ & $8.3 \pm 1.3$ & $<0.00001$ \\
HbA1c $(\%)$ & n.a. & $8.1 \pm 5.5$ & n.a. \\
Diabetes duration $($ years $)$ & & & \\
\hline
\end{tabular}

n.a., not applicable 
Glostrup, Denmark). Urine concentration of sodium was measured by standard biochemical methods.

\section{Statistics}

For normally distributed continuous variables $t$-test was used to compare group mean values, whereas Mann-Whitney test was used otherwise. Chi-square test was used for comparisons of group frequencies. Due to skewed distribution of the Nt-proBNP residuals, Spearman's correlation analysis was used to estimate the correlation between Nt-proBNP and the degree of salt sensitivity. Multiple regression analyses were performed to detect independent effects of various variables on Nt-proANP. As the residuals of the dependent variable (Nt-proANP) were skewly distributed, the natural logarithm of Nt-proANP values $[\ln (\mathrm{Nt}$-proANP) $]$ was used in the analysis. All analyses were performed using an NCSS statistical software (version 6.0.21, Statistical Solutions Limited, Cork, Ireland). Throughout, two-sided tests were used and $P<0.05$ was considered statistically significant.

\section{Results}

Salt sensitivity, Nt-proBNP and Nt-proANP in normotensive subjects with heredity for hypertension

Amongst the normotensive subjects with heredity for hypertension, in whom salt sensitivity was directly measured, the median (interquartile range) concentration of Nt-proBNP at baseline was $173 \mathrm{pmol} \mathrm{L}^{-1}$ (range 140-232). The systolic, diastolic and mean arterial blood pressure, respectively, were $124 \pm 10,77.1 \pm 6.8$ and $92.6 \pm 7.2 \mathrm{mmHg}$ after the low-salt diet and $136 \pm 17,82.1 \pm 7.7$ and $99.9 \pm 10 \mathrm{mmHg}$ after the high-salt diet. The 24-h urinary sodium excretion was $157 \pm 72 \mathrm{mmol}$ at baseline, $10.2 \pm 5.6 \mathrm{mmol}$ after the low-salt diet and $223 \pm 62 \mathrm{mmol}$ after the high-salt diet, indicating good compliance. There was no correlation between Nt-proBNP at baseline and the degree of salt sensitivity $(R=-0.18, P=0.35)$. Before introducing Nt-proANP as a surrogate marker of salt sensitivity in the material of type 2 diabetes patients and nondiabetic subjects with no history of coronary heart disease (Table 1), we extended our previous analysis, which showed a strong direct correlation between Nt-proANP and salt sensitivity in normotensive subjects with heredity for hypertension [5], in order to investigate whether salt sensitivity was the strongest determinant of Nt-proANP independently of potential confounding factors. In a multiple regression analysis with $\ln$ (Nt-proANP) as the dependent variable and the degree of salt sensitivity, age, sex, BMI, heart rate, serum creatinine and systolic and diastolic blood pressure as independent variables, salt sensitivity was directly related $(P=0.00002)$ and heart rate was inversely related $(P=0.02)$ to $\ln (\mathrm{Nt}$-proANP) whereas none of the other variables had any independent effect on $\ln ($ Nt-proANP).

Nt-proANP as a marker of degree of salt sensitivity in type 2 diabetes patients and nondiabetic subjects, without a history of coronary heart disease

Nt-proANP (median, interquartile range) was lower in patients with type 2 diabetes $(505,387-$ $661 \mathrm{pmol} \mathrm{L}^{-1}$ ) than in nondiabetic subjects (536, 421-696 pmol L $\left.{ }^{-1}\right)(P=0.02)$ (Fig. 1). We then performed a multiple regression analysis with $\ln (\mathrm{Nt}-$ proANP) as the dependent variable and diabetes status, age, sex, BMI, cystatin C, heart rate, hypertension treatment and systolic and diastolic blood

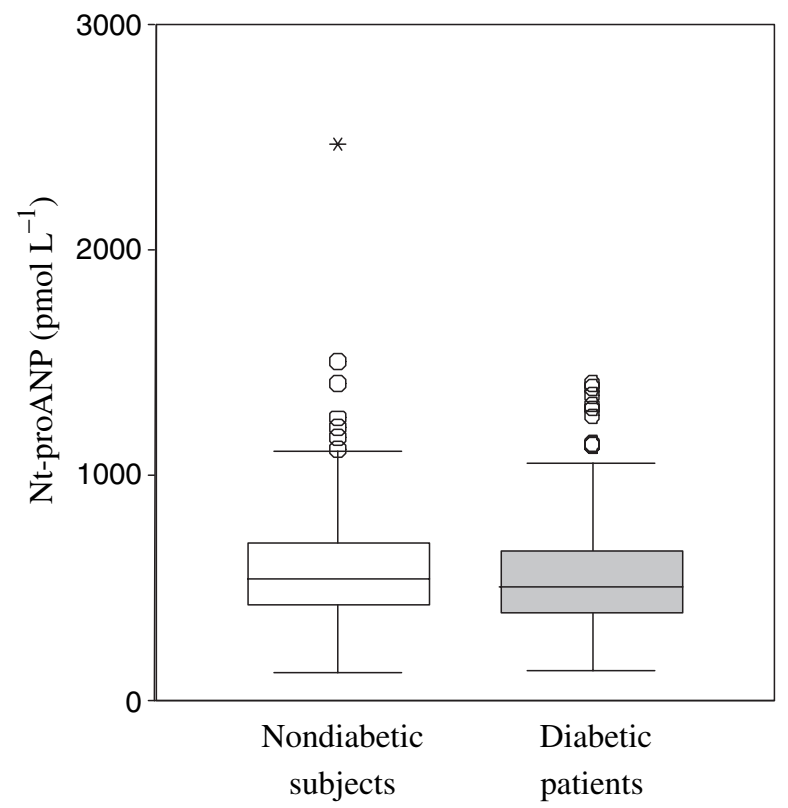

Fig. 1 Box plots of distributions of Nt-proANP in nondiabetic subjects and diabetes patients showing median, interquartile range and range. Circles indicate outliers and ' $*$ ' indicates extreme values. 
Table 2 Multiple regression analysis with $\ln \left(\operatorname{proANP}_{1-30}\right)$ as dependent variable in all subjects and in subjects without antihypertensive treatment

\begin{tabular}{|c|c|c|c|c|}
\hline \multirow[b]{2}{*}{ Independent variables } & \multicolumn{2}{|c|}{ All subjects $(n=483)$} & \multicolumn{2}{|c|}{ Subjects without AHT $(n=352)$} \\
\hline & $\begin{array}{l}\text { Regression } \\
\text { coefficient (SE) }\end{array}$ & $P$-value & $\begin{array}{l}\text { Regression } \\
\text { coefficient (SE) }\end{array}$ & $P$-value \\
\hline Type 2 diabetes $^{\mathrm{a}}$ & $-0.087(0.038)$ & 0.02 & $-0.086(0.041)$ & 0.04 \\
\hline Age (years) & $0.013(0.0028)$ & $<0.00001$ & $0.013(0.0032)$ & 0.00004 \\
\hline Cystatin C $\left(\mu \mathrm{mol} \mathrm{L}{ }^{-1}\right)$ & $0.26(0.075)$ & 0.0006 & $0.19(0.085)$ & 0.03 \\
\hline SBP (mmHg) & $0.0015(0.0011)$ & 0.15 & $0.0017(0.0013)$ & 0.18 \\
\hline DBP (mmHg) & $-0.0047(0.0020)$ & 0.02 & $-0.0050(0.0023)$ & 0.03 \\
\hline $\mathrm{AHT}^{\mathrm{b}}$ & $0.13(0.041)$ & 0.002 & n.a. & n.a. \\
\hline Heart rate $\left(\mathrm{b} \min ^{-1}\right)$ & $-0.0058(0.0012)$ & $<0.00001$ & $-0.0048(0.0013)$ & 0.0003 \\
\hline $\operatorname{Sex}^{\mathrm{c}}$ & $0.096(0.035)$ & 0.006 & $0.11(0.039)$ & 0.004 \\
\hline BMI $\left(\mathrm{kg} \mathrm{m}^{-2}\right)$ & $-0.0035(0.0038)$ & 0.35 & $-0.0044(0.0045)$ & 0.33 \\
\hline
\end{tabular}

AHT, antihypertensive treatment; SE, standard error; SBP, systolic blood pressure; DBP, diastolic blood pressure; BMI, body mass index; n.a., not applicable. ${ }^{\mathrm{a}}$ Absence $(=0)$ or presence $(=1)$ of type 2 diabetes; ${ }^{b}$ absence $(=0)$ or presence $(=1)$ of AHT; ${ }^{c}$ male sex $(=0)$ or female sex $(=1)$. pressure as independent variables. Age, cystatin C, hypertension treatment, and female sex were significantly directly related whereas heart rate, diastolic blood pressure and diabetes status were significantly inversely related to $\ln$ (Nt-proANP) (Table 2). Nt-ProANP (median, interquartile range) was significantly higher in subjects on antihypertensive treatment than in subjects not receiving such treatment amongst nondiabetic subjects (698, 513-841 pmol L ${ }^{-1}$ vs. 522, 415-669 $\mathrm{pmol} \mathrm{L}^{-1}$; $P=0.004)$. Amongst patients with diabetes Nt-ProANP was borderline significantly higher in patients with as compared with patients without antihypertensive treatment $(524, \quad 396-730$ pmol L ${ }^{-1}$ vs. 499 , 371-613 pmol L ${ }^{-1} ; P=0.07$ ). When patients on antihypertensive treatment were excluded, type 2 diabetes patients still had significantly lower Nt-proANP (median, interquartile range) than nondiabetic subjects (499, 371-613 pmol L ${ }^{-1}$ vs. 522, 415-669 pmol L $^{-1}$; $P=0.02)$. In addition, after exclusion of subjects on antihypertensive treatment, the results of the multiple regression analysis remained virtually unchanged with diabetes status as a significant determinant of $\ln$ (Nt-proANP) (Table 2).

\section{Discussion}

\section{Nt-proBNP, Nt-proANP and salt sensitivity}

In contrast to our previous study showing a strong direct relationship between Nt-proANP and salt sensitivity [5], we found no relationship between Nt-proBNP and salt sensitivity in the same material, suggesting that variation of these two peptides, at least in part, reflect different underlying phenotypes. Whereas Nt-proANP seems better as a marker for salt sensitivity, Nt-proBNP and BNP (BNPs) as well as Nt-proANP all seem to be useful in detecting early congestive heart failure [12-14]. In addition, BNPs appear to be good markers of left ventricular hypertrophy [24-26]. In humans Nt-proANP primarily reflects left atrial pressure whereas BNPs are more closely related to left ventricular mass [27]. This is supported by both in vitro and in vivo experimental studies indicating that ANPs reflect the degree of atrial hemodynamic overload whereas brain natriuretic peptides reflect the degree of ventricular hypertrophy [28]. Thus, although echocardiography was not performed in this study, the lack of a relationship between Nt-proBNP and salt sensitivity argues against left ventricular hypertrophy as a major determinant of salt sensitivity. Instead we suggest that apart from, by definition, being a condition characterized by blood pressure changes in response to changes in sodium intake, salt sensitivity is a chronic state of volume expansion leading to elevated Nt-proANP due to increased transmural atrial pressure. Further indications that elevations in Nt-proANP and BNPs reflect partially different underlying phenotypes comes from their opposite relations with plasma renin activity (PRA). Whereas salt sensitivity is accompanied by high NtproANP [5] and low PRA [29], the latter probably reflecting negative feedback to counteract hyperreabsorption of sodium at the level of the renal tubules, left ventricular hypertrophy is associated with high BNPs [24] and high PRA [30]. Thus, 
whereas BNPs seem to be excellent in detecting left ventricular hypertrophy and congestive heart failure [12, 24-26], our present and previous data [5] suggest that Nt-proANP is preferable in estimating the degree of salt sensitivity.

Nt-proANP, as a marker of degree of salt sensitivity, in type 2 diabetes patients and nondiabetic subjects without a history of coronary heart disease

In line with previous studies [31, 32] age, female sex and cystatin $\mathrm{C}$ were directly related, whereas heart rate and diastolic blood pressure were inversely related to Nt-proANP (Table 2). In addition, being on treatment with antihypertensive agents was associated with higher Nt-proANP (Table 2). Despite a number of typical clinical differences between the type 2 diabetes patients and the nondiabetic subjects (Table 1) the diabetic phenotype remained independently associated with lower Nt-proANP in a multiple regression analysis (Table 2). As it is difficult to decide whether the association between high Nt-proANP and treated hypertension (Table 2) is due to an effect of the antihypertensive treatment, hypertension per se, or both, we also performed the analyses after excluding all patients on antihypertensive treatment but the results were virtually unchanged (Table 2). Thus, it is unlikely that the lower Nt-proANP in the diabetes patients was due to confounding effects of any of the clinical variables (Table 1). This is the first study in humans investigating whether presence of type 2 diabetes affects level of the long-acting natriuretic peptide Nt-proANP although elevated levels have been found in the Goto-Kakizaki (GK) rat [33]. A number of small studies have measured ANP and found reduced, elevated or similar levels of this peptide fragment in type 2 diabetes patients as compared with nondiabetic subjects [34-38]. Similarly inconsistent results have been found for the relation between ANP and salt sensitivity [39-44]. It is possible that the natriuretic effects of Nt-proANP itself [7-8] is part of the explanation why this peptide is such a good marker for salt sensitivity. In addition, as a consequence of the rapid break down of ANP after atrial release, ANP circulates in much lower concentrations and is more prone to fluctuations than the relatively stable Nt-proANP molecule $[10,11]$. In this study, the polyclonal antibody preparation used in the radioimmunoassay (RIA) for
Nt-proANP showed $100 \%$ cross reactivity with proANP $_{1-25}$ and thus all peptides, i.e. proANP ${ }_{1-98}$ as well as proteolytic degradation products containing amino acids $1-25$ will be detected in the RIA. The results are reported as molar concentration of Nt-proANP; however, strictly speaking what is measured is equivalents of Nt-proANP immunoreactivity and this may constitute a heterogeneous mix of peptides. These peptides have in common that they originate from proANP ${ }_{1-98}$ and all contain the epitope recognized by the antibody within amino acid segment 1-25. Considering that proANP ${ }_{1-98}$ is secreted in equimolar amounts with the active C-terminal peptide, ANP, measures of N-terminal immunoreactivity may be a better measure of atrial ANP secretion than plasma concentration of ANP itself. We suggest that the inconsistency of previous data concerning the effect of type 2 diabetes on ANP is most likely a consequence of the combination of low number of study subjects and the use of the unstable ANP molecule as the study phenotype. However, it is important to stress that whatever peripheral marker is used to estimate atrial ANP secretion, they are only markers and more exact measures would require kinetic studies with sampling from central blood vessels. In addition, our study cannot distinguish between the effects of atrial secretion and peripheral clearance on Nt-proANP. Although we took into account GFR, as estimated by cystatin $\mathrm{C}$, in our multivariate analyses we cannot exclude that variation in other factors determining Nt-proANP clearance, are affected by the diabetic state.

Based on our previous results showing a strong direct relationship between salt sensitivity and Nt-proANP [5], we used Nt-proANP as a marker of the degree of salt sensitivity. However, as the previous study was performed in nondiabetic subjects some caution in the extrapolation of the data to type 2 diabetes patients is warranted, as we cannot exclude that there is an Nt-proANP suppressing factor, for which we have not adjusted, that is linked to type 2 diabetes but not to low degree of salt sensitivity. However, most of the unmeasured factors that may affect Nt-proANP would be expected to strengthen, rather than to weaken, our main result. For example, it is possible that subclinical cardiovascular disease has been missed in some of our patients. If so, most of the missed cases are likely to belong to the diabetic group as silent cardiovascular disease 
is more common in diabetes patients than in nondiabetic individuals [45]. Although, as discussed above, Nt-proANP is far from a perfect marker of congestive heart failure and ventricular hypertrophy, an overrepresentation of subclinical cardiovascular disease in the diabetes group would be expected to increase the Nt-proANP levels. Thus, if we had been able to adjust for this potential confounder the result of lower Nt-proANP concentrations in type 2 diabetes patients as compared with nondiabetic subjects would most likely be strengthened.

Our finding indicating lower degree of salt sensitivity in type 2 diabetes patients than in nondiabetic subjects may seem surprising as hyperinsulinaemia, a typical feature of the type 2 diabetic patient, has been suggested to cause renal sodium retention and thereby salt-sensitive hypertension [16]. However, our diabetes patients had a mean disease duration of more than 8 years (Table 1). Such a long duration of type 2 diabetes is likely to lead to many different pro-hypertensive processes, other than increased salt sensitivity, thereby attenuating the relative contribution of salt sensitivity to hypertension in the diabetic population. On the other hand, the suggested link between hyperinsulinaemia and salt sensitivity [16] may well be central for the pathogenesis of hypertension in nondiabetic subjects and in the early stages of type 2 diabetes.

In conclusion, patients with type 2 diabetes had lower levels of Nt-proANP than nondiabetic subjects, suggesting that salt sensitivity is reduced in type 2 diabetes. Our finding implies that other pathophysiological mechanisms than salt sensitivity explain the increased prevalence of hypertension in type 2 diabetes. However, the range of Nt-proANP amongst type 2 diabetes patients was wide suggesting that a subset of these patients have a high degree of salt sensitivity and therefore will benefit from dietary salt restriction.

\section{Conflict of interest statement}

There is no conflict of interest in connection with this paper.

\section{Acknowledgements}

This study was supported by grants from the Swedish Medical Research Council, the Swedish Heart and Lung Foundation, the Medical Faculty of Lund
University, Malmö University Hospital, the Albert Påhlsson Research Foundation, the Crafoord Foundation, the Ernhold Lundströms Research Foundation, the Region Skane, the Sigrid Juselius Foundation and the Folkhälsan Research Foundation.

\section{References}

1 European Society of Hypertension-European Society of Cardiology. 2003 European Society of Hypertension-European Society of Cardiology guidelines for the management of arterial hypertension. J Hypertens 2003; 21: 1011-53.

2 Chobanian AV, Bakris GL, Black HR et al. The Seventh Report of the Joint National Committee on Prevention, Detection, Evaluation, and Treatment of High Blood Pressure: the JNC 7 report. JAMA 2003; 289: 2560-72.

3 Sacks FM, Svetkey LP, Vollmer WM et al. Effects on blood pressure of reduced dietary sodium and the Dietary Approaches to Stop Hypertension (DASH) diet. DASH-Sodium Collaborative Research Group. N Engl J Med 2001; 344: 3-10.

4 Mattes RD, Donnelly D. Relative contributions of dietary sodium sources. J Am Coll Nutr 1991; 10: 383-93.

5 Melander O, Frandsen E, Groop L, Hulthen UL. Plasma ProANP(1-30) reflects salt sensitivity in subjects with heredity for hypertension. Hypertension 2002; 39: 996-9.

6 Thibault G, Garcia R, Gutkowska J et al. The propeptide Asn1Tyr126 is the storage form of rat atrial natriuretic factor. Biochem J 1987; 241: 265-72.

7 Vesely DL. Atrial natriuretic hormones originating from the $\mathrm{N}$-terminus of the atrial natriuretic factor prohormone. Clin Exp Pharmacol Physiol 1995; 22: 108-14.

8 Dietz JR, Scott DY, Landon CS, Nazian SJ. Evidence supporting a physiological role for proANP-(1-30) in the regulation of renal excretion. Am J Physiol Regul Integr Comp Physiol 2001; 280: R1510-17.

9 Forssmann WG, Richter R, Meyer M. The endocrine heart and natriuretic peptides: histochemistry, cell biology, and functional aspects of the renal urodilatin system. Histochem Cell Biol 1998; 110: 335-57.

10 Cusson JR, Thibault G, Kuchel O, Hamet P, Cantin M, Larochelle P. Cardiovascular, renal and endocrine responses to low doses of atrial natriuretic factor in mild essential hypertension. J Hum Hypertens 1989; 3: 89-96.

11 Clerico A, Del Ry S, Giannessi D. Measurement of cardiac natriuretic hormones (atrial natriuretic peptide, brain natriuretic peptide, and related peptides) in clinical practice: the need for a new generation of immunoassay methods. Clin Chem 2000; 46: 1529-34.

12 de Lemos JA, McGuire DK, Drazner MH. B-type natriuretic peptide in cardiovascular disease. Lancet 2003; 362: 316-22.

13 Lerman A, Gibbons RJ, Rodeheffer RJ et al. Circulating Nterminal atrial natriuretic peptide as a marker for symptomless left-ventricular dysfunction. Lancet 1993; 341: 1105-9.

14 Daggubati S, Parks JR, Overton RM, Cintron G, Schocken DD, Vesely DL. Adrenomedullin, endothelin, neuropeptide Y, atrial, brain, and C-natriuretic prohormone peptides compared as early heart failure indicators. Cardiovasc Res 1997; 36: 246-55.

15 Tarnow L, Rossing P, Gall MA, Nielsen FS, Parving HH. Prevalence of arterial hypertension in diabetic patients before and after the JNC-V. Diabetes Care 1994; 17: 1247-51. 
16 Reaven GM. The kidney: an unwilling accomplice in syndrome X. Am J Kidney Dis 1997; 30: 928-31.

17 Groop L, Forsblom C, Lehtovirta M et al. Metabolic consequences of a family history of NIDDM (the Botnia study): evidence for sex-specific parental effects. Diabetes 1996; 45: 1585-93.

18 Diabetes mellitus. Report of a WHO Study Group. World Health Organ Tech Rep Ser 1985; 727: 1-113.

19 Isomaa B, Almgren P, Tuomi T et al. Cardiovascular morbidity and mortality associated with the metabolic syndrome. Diabetes Care 2001; 24: 683-9.

20 Vesely DL. Natriuretic peptides and acute renal failure. Am J Physiol Renal Physiol 2003; 285: F167-77.

21 Newman DJ, Thakkar H, Edwards RG et al. Serum cystatin C measured by automated immunoassay: a more sensitive marker of changes in GFR than serum creatinine. Kidney Int 1995; 47: 312-8.

22 Kyhse-Andersen J, Schmidt C, Nordin G et al. Serum cystatin $\mathrm{C}$, determined by a rapid, automated particle-enhanced turbidimetric method, is a better marker than serum creatinine for glomerular filtration rate. Clin Chem 1994; 40: 1921-6.

23 Mussap M, Ruzzante N, Varagnolo M, Plebani M. Quantitative automated particle-enhanced immunonephelometric assay for the routinary measurement of human cystatin C. Clin Chem Lab Med 1998; 36: 859-65.

24 Suzuki M, Yamamoto K, Watanabe S, Iwata T, Hamada M, Hiwada K. Association between elevated brain natriuretic peptide levels and the development of left ventricular hypertrophy in patients with hypertension. Am J Med 2000; 108: 627-33.

25 Yamamoto K, Burnett JC, Jougasaki M et al. Superiority of brain natriuretic peptide as a hormonal marker of ventricular systolic and diastolic dysfunction and ventricular hypertrophy. Hypertension 1996; 28: 988-94.

26 Luchner A, Burnett JC, Jougasaki M et al. Evaluation of brain natriuretic peptide as marker of left ventricular dysfunction and hypertrophy in the population. J Hypertens 2000; 18: 1121-8.

27 Qi W, Mathisen P, Kjekshus J et al. Natriuretic peptides in patients with aortic stenosis. Am Heart J 2001; 142: 725-32.

28 de Bold AJ, Bruneau BG, Kuroski de Bold ML. Mechanical and neuroendocrine regulation of the endocrine heart. Cardiovasc Res 1996; 31: 7-18.

29 Melander O, Frandsen E, Groop L, Hulthen UL. No evidence of a relation between $11 \beta$-hydroxysteroid dehydrogenase type 2 activity and salt sensitivity. Am J Hypertens 2003; 16: $729-$ 33.

30 Malmqvist K, Ohman KP, Lind L, Nystrom F, Kahan T. Relationships between left ventricular mass and the reninangiotensin system, catecholamines, insulin and leptin. J Intern Med 2002; 252: 430-9.

31 Loke I, Squire IB, Davies JE, Ng LL. Reference ranges for natriuretic peptides for diagnostic use are dependent on age, gender and heart rate. Eur J Heart Fail 2003; 5: 599-606.
32 Wang TJ, Larson MG, Levy D et al. Impact of age and sex on plasma natriuretic peptide levels in healthy adults. Am J Cardiol 2002; 90: 254-8.

33 Vesely DL, Gower WR, Jr, Dietz JR et al. Elevated atrial natriuretic peptides and early renal failure in type 2 diabetic Goto-Kakizaki rats. Metabolism 1999; 48: 771-8

34 Shin SJ, Lee YJ, Hsiao PJ, Tsai JH. Increased urinary atrial natriuretic peptide-like immunoreactivity excretion but decreased plasma atrial natriuretic peptide concentration in patients with hyperosmolar-hyperglycemic nonketotic syndrome. Diabetes Care 1999; 22: 1181-5.

35 Nannipieri M, Seghieri G, Catalano C, Prontera T, Baldi S, Ferrannini E. Defective regulation and action of atrial natriuretic peptide in type 2 diabetes. Horm Metab Res 2002; 34: 265-70.

36 Yano Y, Ura H, Gabazza EC et al. Circulating levels of $7 \mathrm{~S}$ domain of type IV collagen and atrial natriuretic peptide in normotensive type 2 diabetic patients with or without retinopathy. Horm Metab Res 1998; 30: 103-7.

37 Yano Y, Gabazza EC, Katsuki A et al. Plasma levels of natriuretic peptides are correlated with renin activity in normotensive type 2 diabetic patients. Diabetes Care 2000; 23: 1853-5.

38 Chattington PD, Anderson JV, Rees LH, Leese GP, Peters JR, Vora JP. Atrial natriuretic peptide in type 2 diabetes mellitus: response to a physiological mixed meal and relationship to renal function. Diabet Med 1998; 15: 375-9.

39 Kohno M, Yasunari K, Murakawa K, Kanayama Y, Matsuura T, Takeda T. Effects of high-sodium and low-sodium intake on circulating atrial natriuretic peptides in salt-sensitive patients with systemic hypertension. Am J Cardiol 1987; 59: 1212-3.

40 Gerdts E, Myking OL, Omvik P. Salt sensitive essential hypertension evaluated by 24 hour ambulatory blood pressure. Blood Press 1994; 3: 375-80.

41 McKnight JA, Roberts G, Sheridan B, Atkinson AB. The effect of low and high sodium diets on plasma atrial natriuretic factor, the renin-aldosterone system and blood pressure in subjects with essential hypertension. Clin Endocrinol 1994; 40: 73-7.

42 Ferri C, Bellini C, Carlomagno A, Perrone A, Santucci A. Urinary kallikrein and salt sensitivity in essential hypertensive males. Kidney Int 1994; 46: 780-8.

43 de la Sierra A, Lluch MM, Coca A et al. Fluid, ionic and hormonal changes induced by high salt intake in salt-sensitive and salt-resistant hypertensive patients. Clin Sci 1996; 91: 155-61.

44 Campese VM, Tawadrous M, Bigazzi R et al. Salt intake and plasma atrial natriuretic peptide and nitric oxide in hypertension. Hypertension 1996; 28: 335-40.

45 Laakso M. Hyperglycemia and cardiovascular disease in type 2 diabetes. Diabetes 1999; 48: 937-42.

Correspondence: Olle Melander, Department of Endocrinology, Malmö University Hospital MAS, S-205 02 Malmö, Sweden. (fax: +46 40 337042; e-mail: olle.melander@endo.mas.lu.se). 